\title{
On-orbit calibration of the Geosynchronous Imaging Fourier Transform Spectrometer (GIFTS)
}

\author{
R. O. Knuteson ${ }^{\mathrm{a}}$, F. A. Best ${ }^{\mathrm{a}}$, G. E. Bingham ${ }^{\mathrm{b}}$, J. D. Elwell ${ }^{\mathrm{b}}$, H. E. Revercomb ${ }^{\mathrm{a}}$, \\ D. C. Tobin ${ }^{\mathrm{a}}$, D. K. Scott ${ }^{\mathrm{b}}$, J. Taylor ${ }^{\mathrm{a}}$, and W. L. Smith ${ }^{\mathrm{c}}$ \\ ${ }^{a}$ University of Wisconsin-Madison, Madison, WI \\ ${ }^{\mathrm{b}}$ Utah State University, Logan, UT \\ ${ }^{c}$ Hampton University, Hampton, VA
}

\begin{abstract}
The Geosynchronous Imaging Fourier Transform Spectrometer (GIFTS) sensor has been designed to provide highly accurate radiometric and spectral radiances in order to meet the requirements of remote sensing of atmospheric motion from a geostationary orbit. The GIFTS sensor was developed under NASA New Millenium Program funding to demonstrate the tracking of infrared water vapor features in the atmosphere with high vertical resolution. A calibration concept has been developed for the GIFTS instrument design which meets the top level requirement to measure brightness temperature to better than $1 \mathrm{~K}$. The in-flight radiometric calibration is performed using views of two on-board blackbody sources along with cold space. For the GIFTS design, the spectral calibration is established by the highly stable diode laser used as the reference for interferogram sampling, and verified with comparisons to atmospheric absorption line positions. The status of the GIFTS on-orbit calibration approach is described and accuracy estimates are provided. GIFTS is a collaborative activity among NASA Langley Research Center, Utah State Space Dynamics Laboratory, and the University of Wisconsin Space Science and Engineering Center.
\end{abstract}

Keywords: Imaging, FTS, Radiometric, Calibration, Blackbody, Remote Sensing

\section{INTRODUCTION}

The U.S. National Oceanic and Atmospheric Administration (NOAA) operates geostationary operational environmental satellites (GOES) for short-range warning and nowcasting, and polar-orbiting environmental satellites (POES) for longer term forecasting. GOES satellites provide continuous monitoring from space in a geosynchronous orbit about 35,800 km (22,300 miles) above the Earth. A new generation of sensors is under development that will greatly increase the horizontal, vertical, and temporal sampling of the GOES sounder and provide a truly four-dimensional view of the Earth's atmosphere. NOAA's plan for a Hyperspectral Environmental Suite (HES) calls for the replacement of the current GOES instrumentation starting as early as $2013^{1,2}$. NASA's Geosynchronous Imaging Fourier Transform Spectrometer (GIFTS) sensor, under fabrication at the Utah State Space Dynamics Laboratory (SDL), will serve as a valuable test bed for the evaluation of approaches to flight hardware and ground data processing in the years leading up to NOAA's operational Hyperspectral Environmental Suite. The GIFTS sensor makes use of a 2-D array of detectors to increase area coverage rates while providing dramatically higher vertical resolution by measuring the thermal infrared upwelling emission spectrum at high spectral resolution. The sensor calibration makes use of two internal high precision blackbody references in addition to an external view to space. The GIFTS ground data processing algorithms used to convert from instrument values (Level 0 data) to geo-located, calibrated radiances (Level 1 data) are under development at the University of Wisconsin Space Science and Engineering Center (UW-SSEC).

The UW-SSEC has a long history of development in support of the meteorological application of geostationary observations with an emphasis on the determination of atmospheric winds by the tracking of radiance features caused by the motion of clouds and water vapor. The development of the spin scan cloud camera in the early 1960s lead to the first global images from geostationary orbit as shown in Fig. 1. The UW-SSEC also developed the science algorithms and software for the processing of this geostationary satellite data stream (see Fig. 2). The animation of a time series of geostationary images was used to track the motion of atmospheric features. The Mancomputer Interactive Data Access System (McIDAS) was developed at UW-SSEC in the 1970s and 1980s and has

Copyright 2004 Society of Photo-Optical Instrumentation Engineers.

This paper was published in the proceedings of the SPIE, Fourth International Asia-Pacific Environmental Remote Sensing Symposium, 8 November 2004; Honolulu, Hawaii, and is made available as an electronic preprint with permission of SPIE. One print may be made for personal use only. Systematic or multiple reproduction, distribution to multiple locations via electronic or other means, duplication of any material in this paper for a fee or for commercial purposes, or modification of the content of the paper are prohibited. 
been used ever since in operational data processing of GOES satellites by NOAA. The GIFTS/HES concept is the natural next step in the increasingly sophisticated exploitation of weather observations from geostationary orbit by providing enhanced vertical resolving capability to complement the high spatial and temporal sampling.
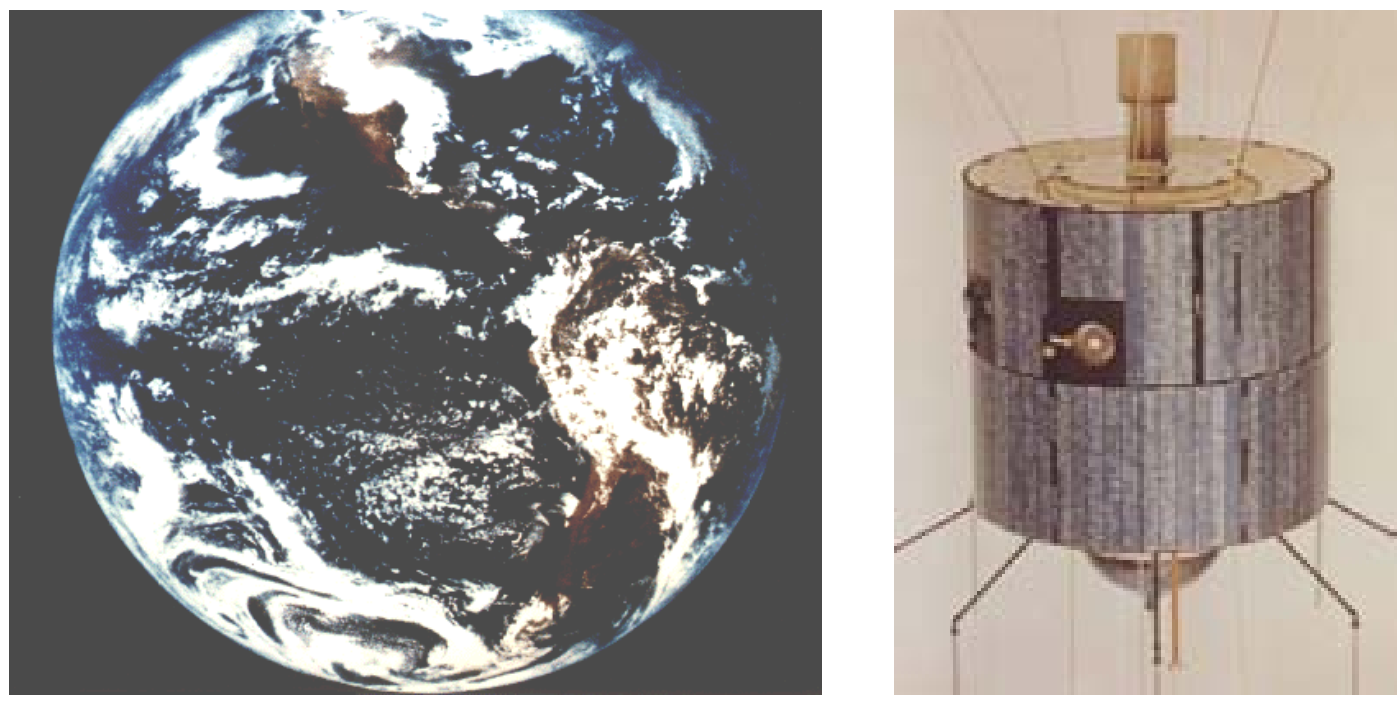

Figure 1. University of Wisconsin involvement in geostationary weather satellites dates back to the invention of the "spin scan cloud camera” by the founding director of the Space Science and Engineering Center, Prof. Verner Suomi, in collaboration with Prof. Robert Parent. The color full disk Earth image (left) shown was taken by ATS-3 (right) in 1966. Prof. Suomi's promotion of the use of geosynchronous orbit for tracking the motion of clouds to obtain winds was captured in his famous phrase, "The clouds move, not the satellite!” The GIFTS/HES concept is the natural technological follow-on to this goal by allowing the time dependent motion tracking of atmospheric constituents, e.g. water vapor, at many more atmospheric levels in the vertical than was previously possible.

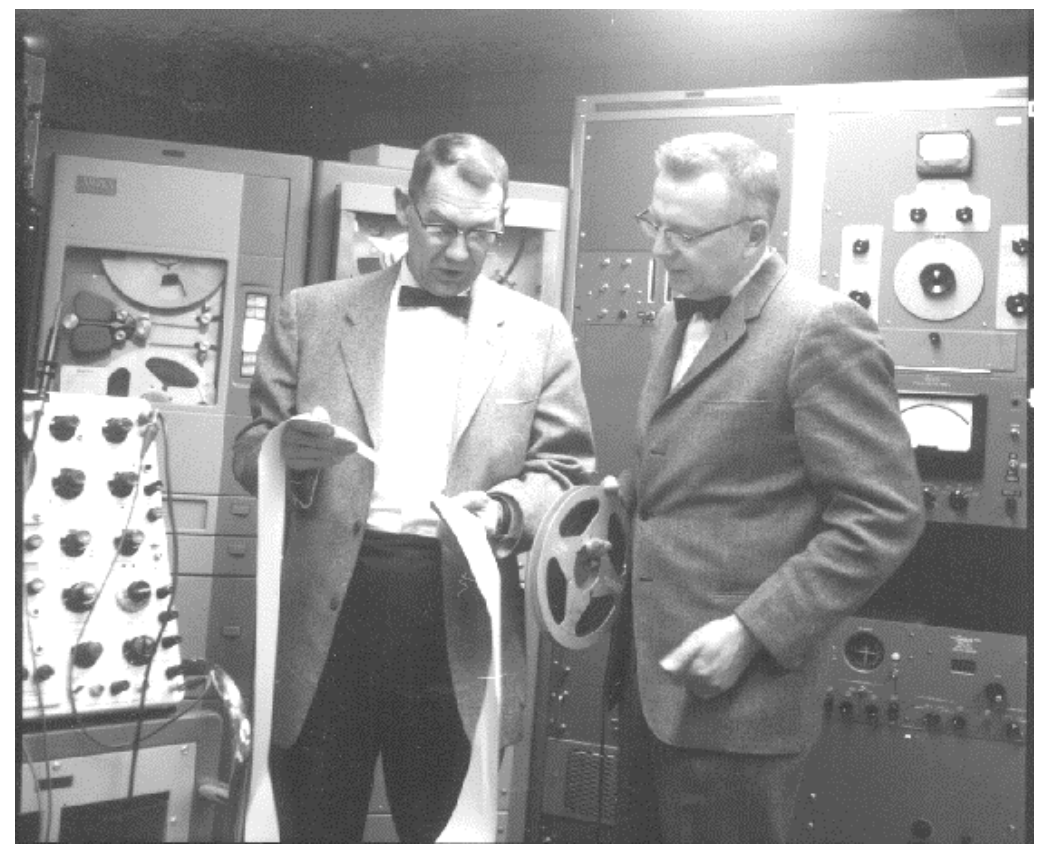

Figure 2. University of Wisconsin involvement in the development of the hardware and software required to make images of the spin scan camera data lead to the development of the Man-computer Interactive Data Access System (McIDAS) which, after many generations, is still used extensively by NOAA in the processing of geostationary satellite imagery. Prof. Robert Parent (left) and Prof. Verner Suomi (right) are shown analyzing meteorological data using an early ground processing system (circa

Copyright 2004 Society of Photo-Optical Instrumentation Engineers.

This paper was published in the proceedings of the SPIE, Fourth International Asia-Pacific Environmental Remote Sensing Symposium, 8 November 2004; Honolulu, Hawaii, and is made available as an electronic preprint with permission of SPIE. One print may be made for personal use only. Systematic or multiple reproduction, distribution to multiple locations via electronic or other means, duplication of any material in this paper for a fee or for commercial purposes, or modification of the content of the paper are prohibited. 
1959). The processing of large amounts of satellite data continue to pose a challenge for the future hyper-spectral sounders which have both high spatial and high temporal resolution.

\section{BACKGROUND}

The UW-SSEC pioneered the development of absolute radiometric and spectral calibration for "warm" InfraRed Fourier Transform Spectrometers (FTIR) with an accuracy and reproducibility that is sufficient for the use of the observations in atmospheric remote sensing ${ }^{3}$. Even though the GIFTS is a "cold" instrument, the same physical principles developed for the UW-SSEC instruments (and adopted by the NPOESS Crosstrack IR Sounder program) will be applied to the calibration of the GIFTS radiances in order to take into account gain and offset changes in the instrument during normal operation. The GIFTS spectral coverage indicated in Fig. 3 illustrates that the dynamic range of signals from terrestrial thermal infrared radiation spans hundreds of degreees. However, the demands of remote sensing of atmospheric effects are high since the signal of subtle changes in temperature and atmospheric humidity from the mean atmospheric state are only tenths of degrees ${ }^{4}$. Achieving absolute calibration at the tenth of degree accuracy level is a goal that is within the reach of high spectral resolution IR remote sensing using precision on-board blackbody references with NIST traceability. This approach has been demonstrated at the UW-SSEC in both the groundbased Atmospheric Emitted Radiance Interferometer (AERI) program and the aircraft-based Highresolution Interferometer Sounder (HIS) program. ${ }^{5,6,7}$

In both the AERI and HIS programs, FTIR spectrometers have been used in order to take advantage of the very high spectral frequency knowledge that is inherent in the FTS design. At the relatively high spectral resolutions (resolving power $>1000$ ) in the thermal infrared, the wavenumber sampling and instrument line shapes must be known to better than $1 \%$ accuracy or errors will be introduced in the comparison with forward model calculations that exceed the radiometric requirement. With an FTIR sensor, a single parameter determines the wavenumber sampling of each spectral band and all the spectral elements see the same field of view on the Earth. The FTS spectral parameter can be determined pre-launch but also in-flight by comparison to known spectral absorption lines across the spectral band of interest. In contrast, a grating spectrometer that uses individual detector elements at each spectral element requires extensive pre-launch testing to characterize the many individual unique spectral response function (SRF) shapes. Unfortunately the grating SRFs are impossible to confirm on-orbit to the desired accuracy. The excellent spectral knowledge and stability of the FTIR system was the primary motivation for the selection of FTS for the GIFTS sensor. The grating spectrometer implemented on the NASA Atmospheric InfraRed Sounder (AIRS) sensor has the further disadvantage that each detector has a slightly different field of view to the ground. This leads to spectral "artifacts" when viewing scenes that are not uniform temperature (over a $15 \mathrm{~km}$ region). This is illustrated in Fig. 4 where spectral discontinuities are obvious in observations from the NASA AIRS grating spectrometer over tropical storm Isador, rendering the data effectively unusable near the eye of the storm. The GIFTS sensor avoids this problem by using a single detector to measure all the wavelengths of a spectral band simultaneously for each individual field of view to the Earth.

The ability to accurately calibrate high spectral resolution infrared observations is also important for the future of detecting global change from space-borne observations ${ }^{8}$. The technology exists with precision blackbodies and FTS laser spectral sampling to approach the tenth of degree accuracy and stability desired for the detection of global climate change on decadal scales. Although this is outside the scope of the GIFTS sensor requirements, the GIFTS design shows the feasibility of high absolute accuracy in a practical implementation for sensors in geostationary orbit. The design makes use of two high precision cavity radiometers with high absolute emissivity $(>0.998)$ and good long term stability (diffuse paints). The cavity blackbodies used for GIFTS are built and calibrated at the UWSSEC based upon heritage with the AERI and HIS programs. The unique approach for GIFTS is to place these reference cavities aft of the Earth viewing telescope with an "on-demand" flip in mirror to direct the IR emission from the blackbodies into the sensor. The main advantage of this approach is that the IR beam is much smaller after the telescope so that a true high emissivity cavity design can be used for the blackbodies while keeping the volume, weight, and power requirements to a minimum. The successful design of these blackbodies for the GIFTS sensor is described in ${ }^{9}$. The high absolute accuracy of the onboard reference blackbodies compensates for the additional uncertainty from degradation of the telescope optics over time. A scheme for monitoring this telescope degradation over time that makes use of the internal reference views and views to deep space has been devised for the GIFTS sensor by the Utah State University Space Dynamics Laboratory. ${ }^{10}$

Copyright 2004 Society of Photo-Optical Instrumentation Engineers.

This paper was published in the proceedings of the SPIE, Fourth International Asia-Pacific Environmental Remote Sensing Symposium, 8 November 2004; Honolulu, Hawaii, and is made available as an electronic preprint with permission of SPIE. One print may be made for personal use only. Systematic or multiple reproduction, distribution to multiple locations via electronic or other means, duplication of any material in this paper for a fee or for commercial purposes, or modification of the content of the paper are prohibited. 


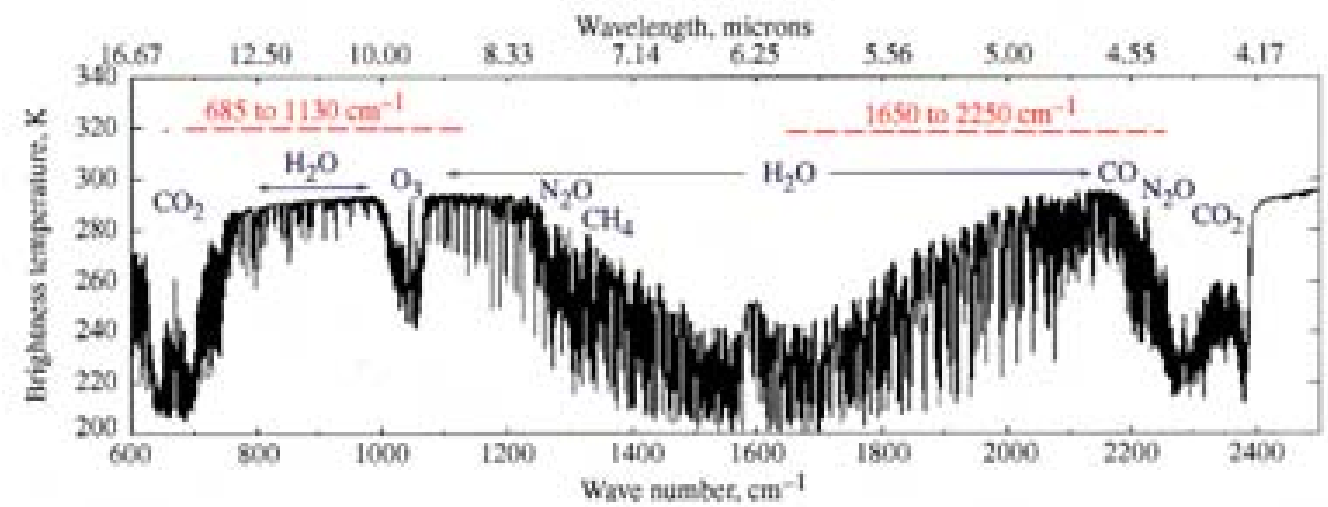

Figure 3. A calculation of the top of atmosphere radiance emitted by the standard atmosphere in units of equivalent brightness temperature (Kelvin). The dashed lines indicate the two spectral bands selected for the NASA GIFTS sensor. The longwave (LW) band covers the traditional "temperature sounding" region for the characterization of atmospheric temperature from the top of the atmosphere to the surface and includes the 8-12 $\mu \mathrm{m}$ IR window for the characterization of land surface and cloud top temperature and emissivity. The shortwave/midwave (SWM) band includes a non-traditional coverage of the shortwave side of the "6.3 $\mu \mathrm{m}$ water vapor sounding" region. The short-midwave band coverage $\left(1650-2250 \mathrm{~cm}^{-1}\right)$ was shown by analysis to be optimal for three reasons; 1) this region avoids the interference of "fixed" gases $\mathrm{N}_{2} \mathrm{O}$ and $\mathrm{CH}_{4}$ which degrade the water vapor sounding performance, 2) the shorter wavelength (fewer thermal photons) leads to better signal to noise performance for the detectors chosen, 3 ) provides coverage of carbon monoxide thereby allowing the tracking of air pollution plumes from source to sink.

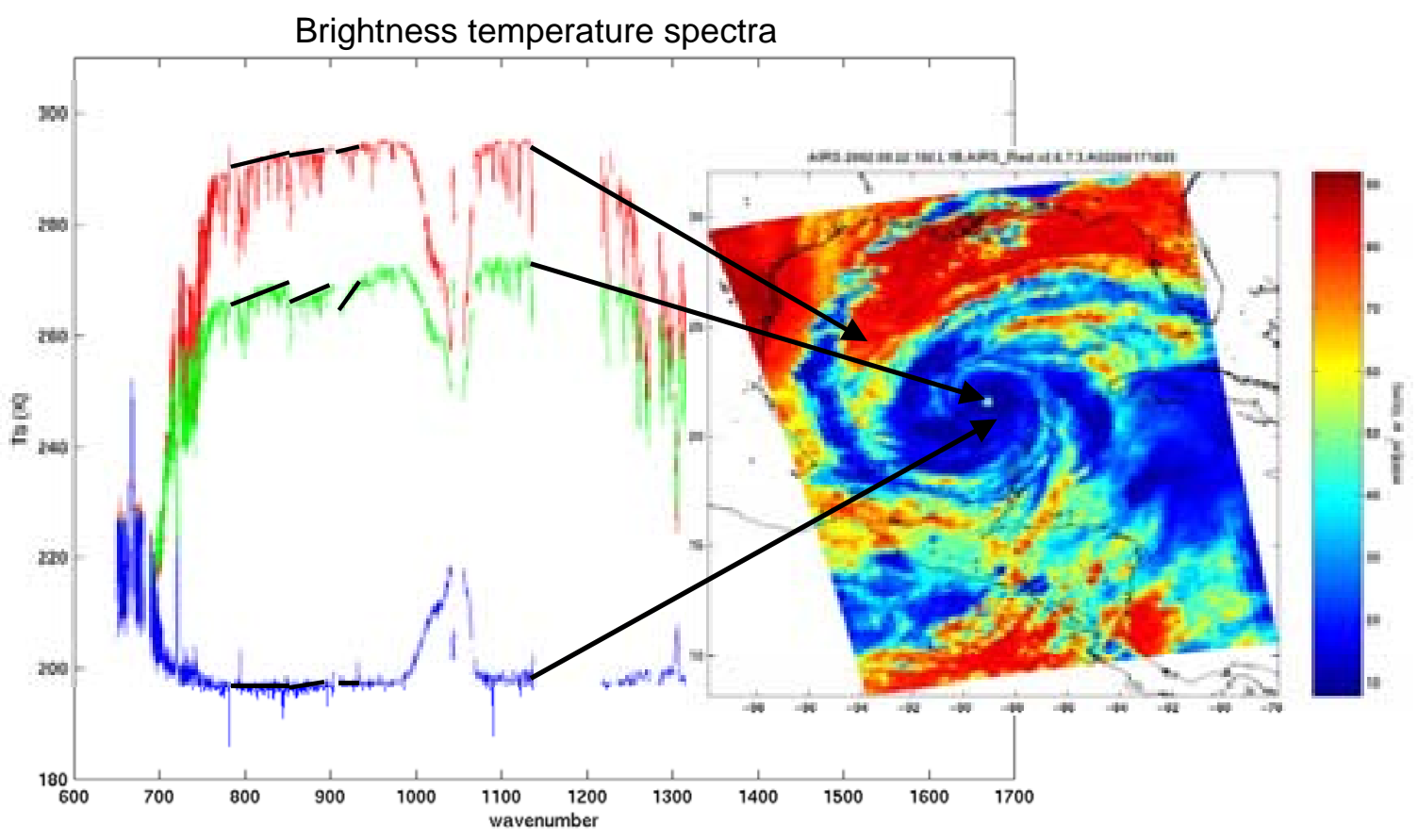

Figure 4. Spectral artifacts in the 8-12 $\mu \mathrm{m}$ window region from a grating spectrometer are obvious from this observation from the NASA AIRS sensor on the Aqua platform. The AIRS observation that includes the eye of tropical storm Isadore (22 Sept 2002 19:12-19:18 UTC) has large discontinuities (indicated by the black bars). This is not a physical atmospheric effect, rather it is caused by the fact that the thousands of individual AIRS detectors that record each spectral element have thousands of different fields of view to the earth. This is a serious problem for the use of this data in scenes of mixed cloudiness. Notice that this spectral artifact disappears for fields of view that have little temperature contrast (either entirely warm or entirely cold). The FTIR design avoids this problem by using a single detector to record all the wavelengths in a spectral band simultaneously and an on-board metrology laser sampling system to provide a consistent wavenumber scale across each spectral band.

Copyright 2004 Society of Photo-Optical Instrumentation Engineers.

This paper was published in the proceedings of the SPIE, Fourth International Asia-Pacific Environmental Remote Sensing Symposium, 8 November 2004; Honolulu, Hawaii, and is made available as an electronic preprint with permission of SPIE. One print may be made for personal use only. Systematic or multiple reproduction, distribution to multiple locations via electronic or other means, duplication of any material in this paper for a fee or for commercial purposes, or modification of the content of the paper are prohibited. 


\section{GIFTS RADIOMETRIC CALIBRATION}

The GIFTS radiometric calibration is designed to use two small reference blackbodies located behind the telescope, combined with a space view ${ }^{11}$. Figure 5 is a schematic of the GIFTS optical design showing the location of the two internal blackbody reference sources between the optical elements labeled M2 and $\mathrm{M}^{12}{ }^{12}$. This is also the location of an image of the aperture stop for each detector in the focal plane arrays. Figure 6 illustrates the "flip mirror" mechanism with a linear slide to position either the warm or cold blackbody into the instrument beam. The blackbody design is scaled from the UW ground-based design used on AERI and S-HIS aircraft instruments. Constraints on the original spacecraft envelope prevented a traditional external large aperture blackbody implementation. The advantages of using two internal blackbodies compared to one large external blackbody include;

(1) higher emissivity is practical with small size,

(2) effective temperature of the body easier to characterize, and

(3) protection from solar forcing gradients.

A photo of the GIFTS engineering model blackbody cavity built by UW-SSEC is shown in Figure 6 (Best et al., 2004).

Radiometric calibration of the GIFTS spectrometer has the same considerations as that of any radiometer. Key factors include the accuracy of onboard references, thermal stability over calibration cycles, and linearity after correction. This approach was proven 15 years ago with a warm interferometer by UW-SSEC using the HIS aircraft instrument as described $i^{3}$ and is being used for processing the CrIS data for NPOESS. The cold spectrometer design places a tight constraint on blackbody emissivity uncertainty since the energy reflected from a cold instrument is very small compared to an instrument that is close to the blackbody temperature. This, coupled with volume and mass constraints for GIFTS led to using small high-emissivity internal blackbody references, plus a space view. The broad spectral coverage from a single detector (inherent to FTS) prevents detector-to-detector FOV variations from altering spectral radiance shapes (greatly simplifying FOV co-alignment and testing requirements). The calibration method is summarized in Eq. 1 . This is a modified version of the Revercomb ${ }^{3}$ equation to include the ratio of the transmission of the flip in mirror (labeled " $m$ ") to the fore optics telescope (labeled " $\mathrm{t}$ ").

$$
N=\left(\frac{\tau_{m}}{\tau_{t}}\right)\left(B_{H}-B_{C}\right) \operatorname{Re}\left(\frac{C_{E}-C_{S}}{C_{H}-C_{C}}\right)+B_{S}
$$

The radiance $N$ is derived from raw spectra of Earth $\left(C_{E}\right)$, Space $\left(C_{S}\right)$, and the internal Hot $\left(C_{H}\right)$ and Cold $\left(C_{C}\right)$ blackbodies where $B_{i}$ is the predicted radiance from the Hot, Cold, and Space references including the effective emissivity of the blackbody cavity and the energy reflected off the blackbody from the environment.

The GIFTS calibration requirement has been flown down to requirements on the subsystem components, in particular to the blackbody subsystem. Figure 7 is a block diagram showing the top level GIFTS calibration error budget broken into two main contributions; radiometric and spectral calibration contributions. Each box in these charts contains three pieces of information; the requirement (either from the GIFTS Instrument Requirements Document or an equivalent implied requirement), the budget allocation for that box, and the engineering current best estimate for that item. For example the Radiometric Requirement (labeled 1.1 in Fig. 7) has a requirement of $<1 \mathrm{~K}$, a budget allocation of $<0.9 \mathrm{~K}$, and an engineering best estimate of $<0.73 \mathrm{~K}(\mathrm{LW})$ and $<0.54 \mathrm{~K}$ (SMW). The engineering best estimate for the Radiometric Requirement flows up from the lower level contributions shown in Figure 8. In particular, the calibration budget contribution from uncertainties in the blackbodies is shown as box 1.1.1. The blackbody calibration contribution budget allocation is $<0.5 \mathrm{~K}$ and the engineering best estimates are $<0.35 \mathrm{~K}$ (LW) and $<0.20 \mathrm{~K}$ (SMW). The uncertainty estimates of the UW-SSEC blackbodies are described in much greater detail in ${ }^{9}$. The uncertainty estimates in the calibrated radiance are obtained through a perturbation analysis of Eq. 1 where the uncertainties of the blackbody emissivity and temperature are taken into account. The result of this analysis for the LW and SMW bands is shown in Figs. 9 and 10 as a function of scene temperature. Also shown are the overall GIFTS requirement and an estimate assuming an external blackbody reference with lower emissivity and larger temperature uncertainty. The parameter values used in the uncertainty analysis can be found in Table 1. A similar analysis showing the contribution of spectral calibration uncertainties to the overall calibration budget is included in the next section.

Copyright 2004 Society of Photo-Optical Instrumentation Engineers.

This paper was published in the proceedings of the SPIE, Fourth International Asia-Pacific Environmental Remote Sensing Symposium, 8 November 2004; Honolulu, Hawaii, and is made available as an electronic preprint with permission of SPIE. One print may be made for personal use only. Systematic or multiple reproduction, distribution to multiple locations via electronic or other means, duplication of any material in this paper for a fee or for commercial purposes, or modification of the content of the paper are prohibited. 


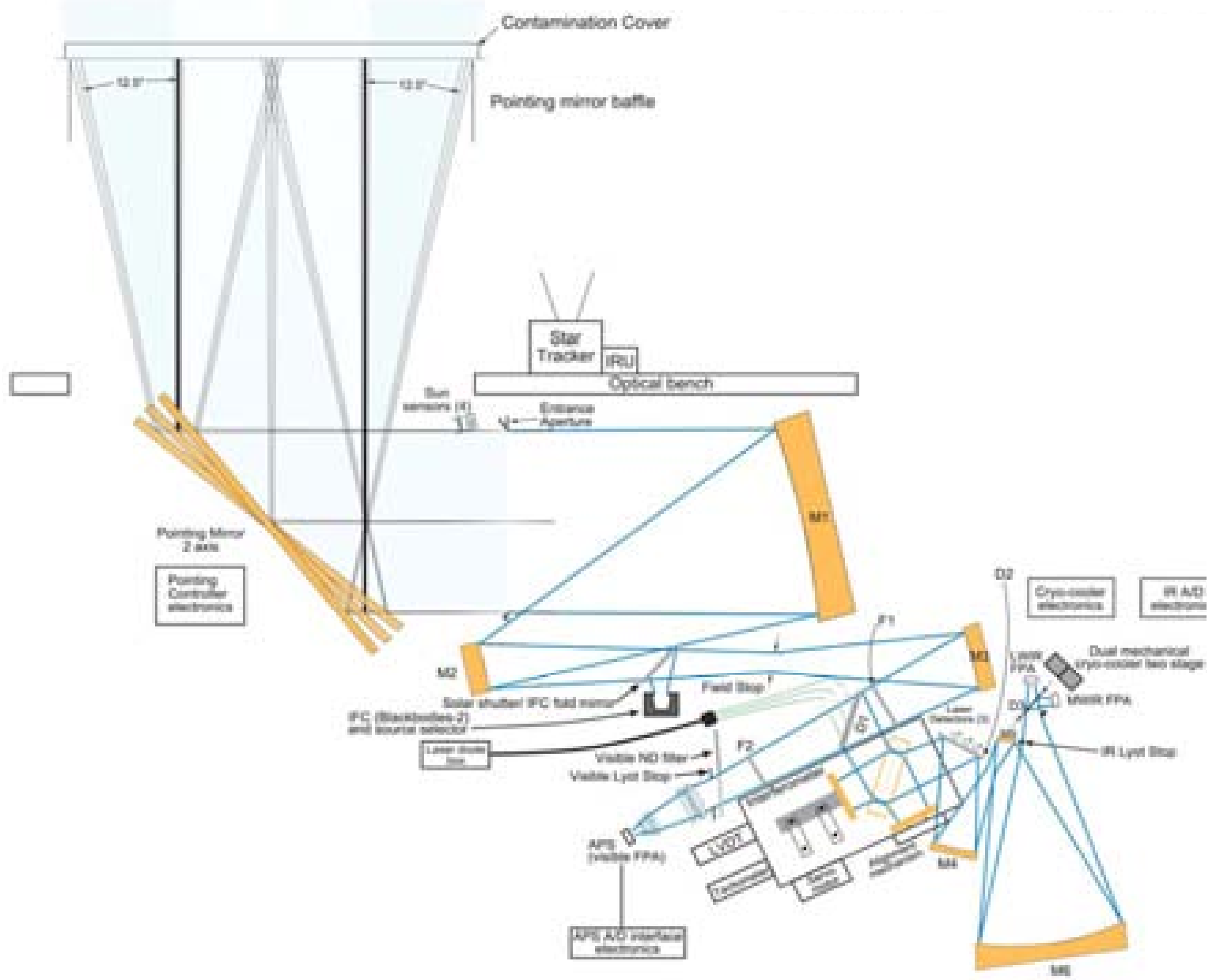

Figure 5. GIFTS sensor module electro-optical functional diagram showing the location of the two UW-SSEC blackbodies (labeled IFC in the diagram) aft of the Earth viewing telescope and the IFC fold mirror which directs energy from the blackbodies into the instrument upon command. Since the beam diameter at this location is small, the internal blackbodies can have high emissivity while remaining relatively compact and lightweight. The blackbodies were designed, built, and calibrated at the UW-SSEC using standards traceable to NIST.
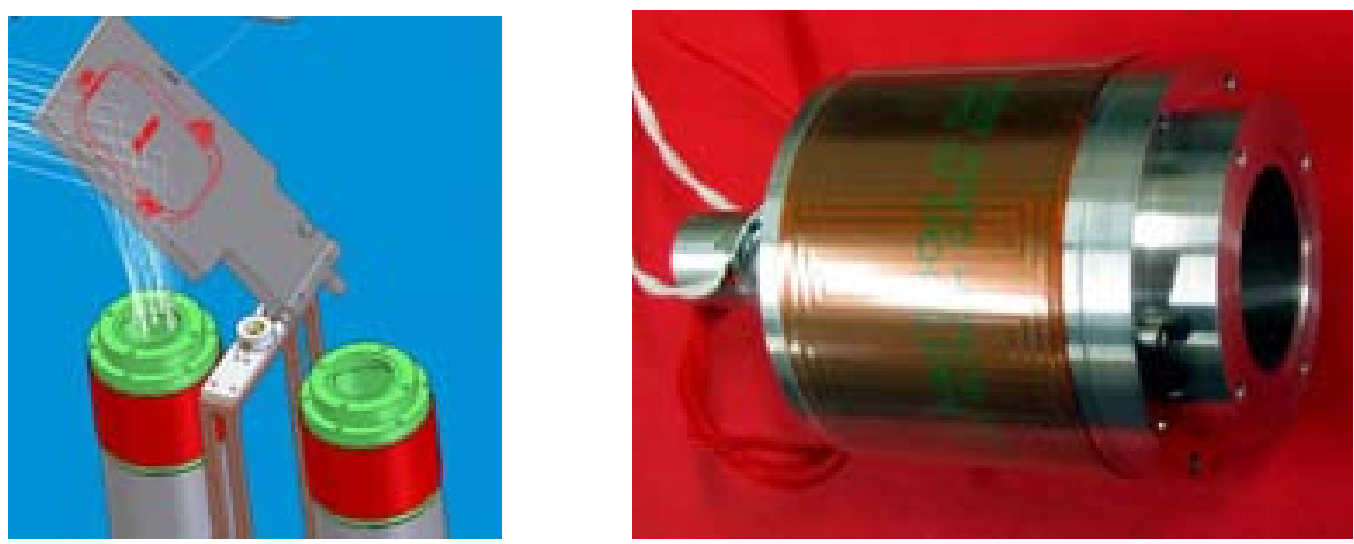

Figure 6. The left-hand panel illustrates two blackbodies and a visible flood source mounted on the same linear slide. One source at a time is correctly positioned under the flip-in mirror. The right-hand illustration is a photo of the UW-SSEC engineering model of the GIFTS blackbody. The blackbody aperture is 1.00 inches in diameter, the width of the cylindrical body is 1.76 inches in diameter, and the total cavity depth is 3.06 inches.

Copyright 2004 Society of Photo-Optical Instrumentation Engineers.

This paper was published in the proceedings of the SPIE, Fourth International Asia-Pacific Environmental Remote Sensing Symposium, 8 November 2004; Honolulu, Hawaii, and is made available as an electronic preprint with permission of SPIE. One print may be made for personal use only. Systematic or multiple reproduction, distribution to multiple locations via electronic or other means, duplication of any material in this paper for a fee or for commercial purposes, or modification of the content of the paper are prohibited. 


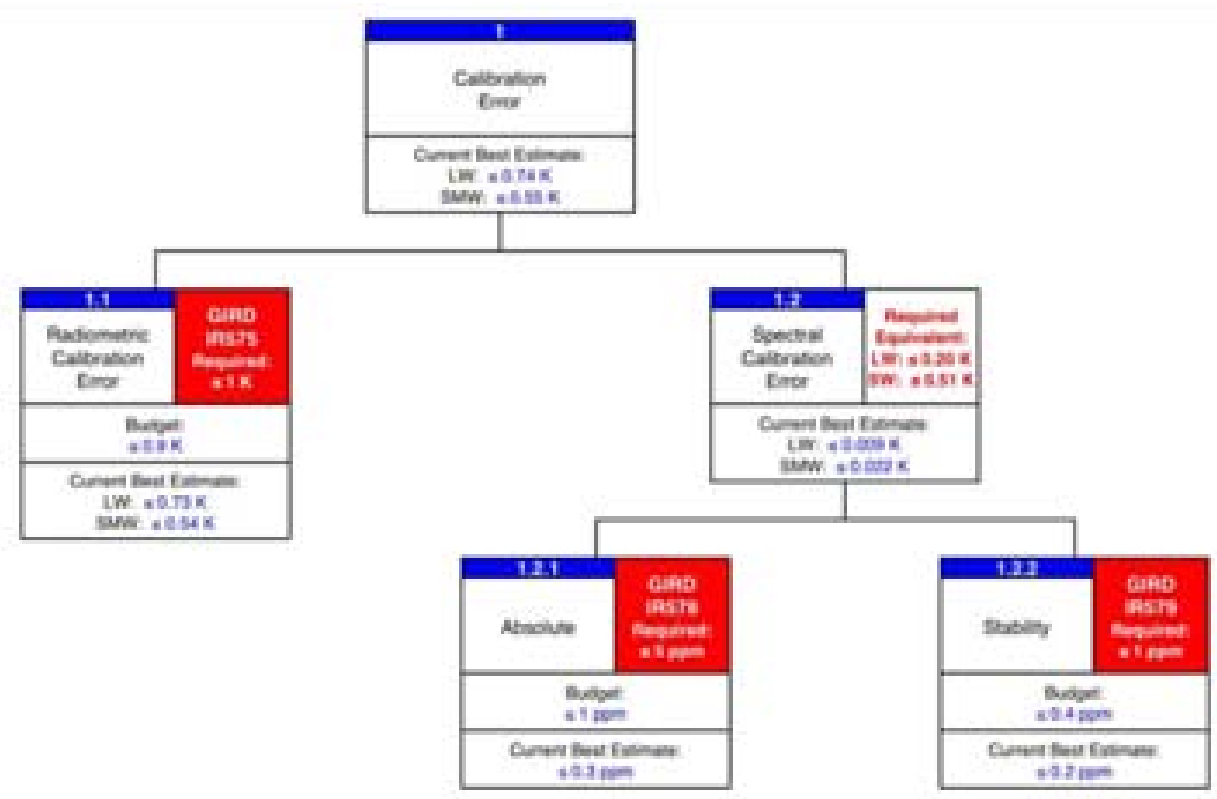

Figure 7. GIFTS top-level absolute calibration budget stating the radiometric and spectral requirements, the error budget allocation, and the current engineering best estimates.

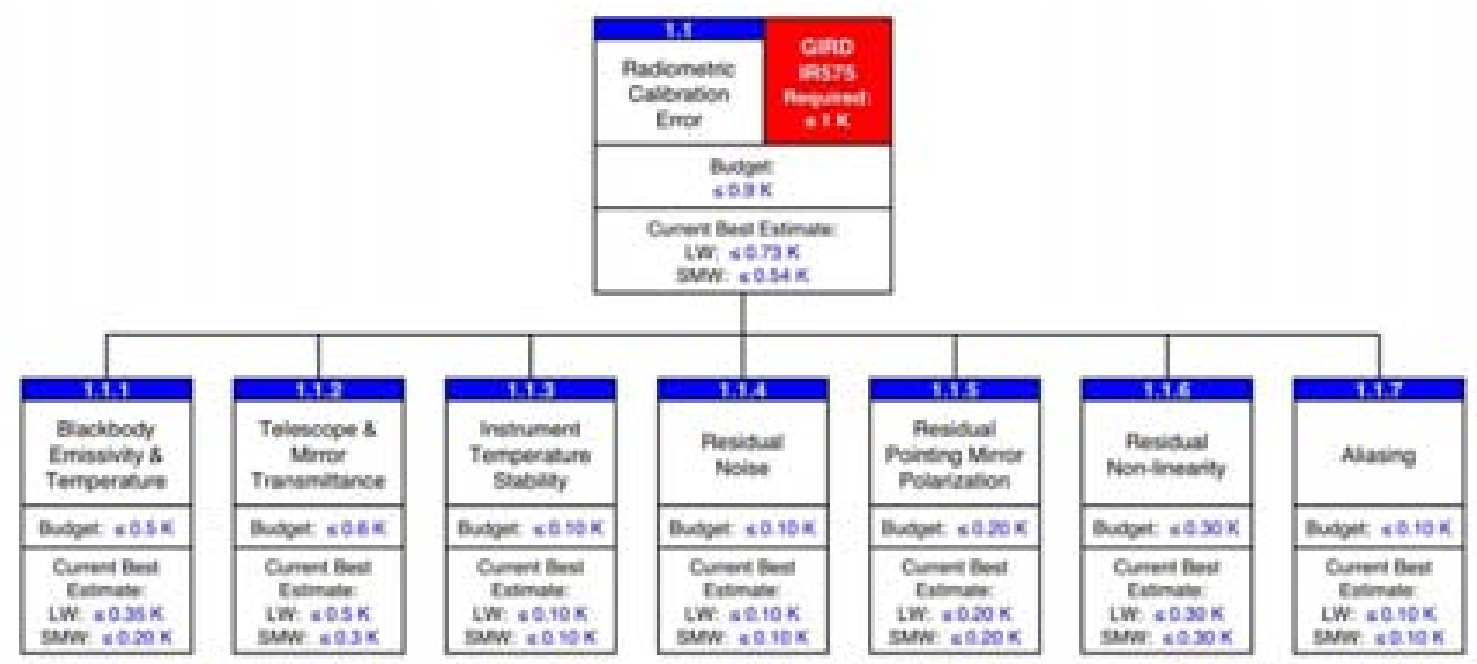

Figure 8. GIFTS radiometric calibration budget. The engineering estimates of the individual error contributors are combined (RSS) to obtain the total 3-sigma calibration error estimate. 


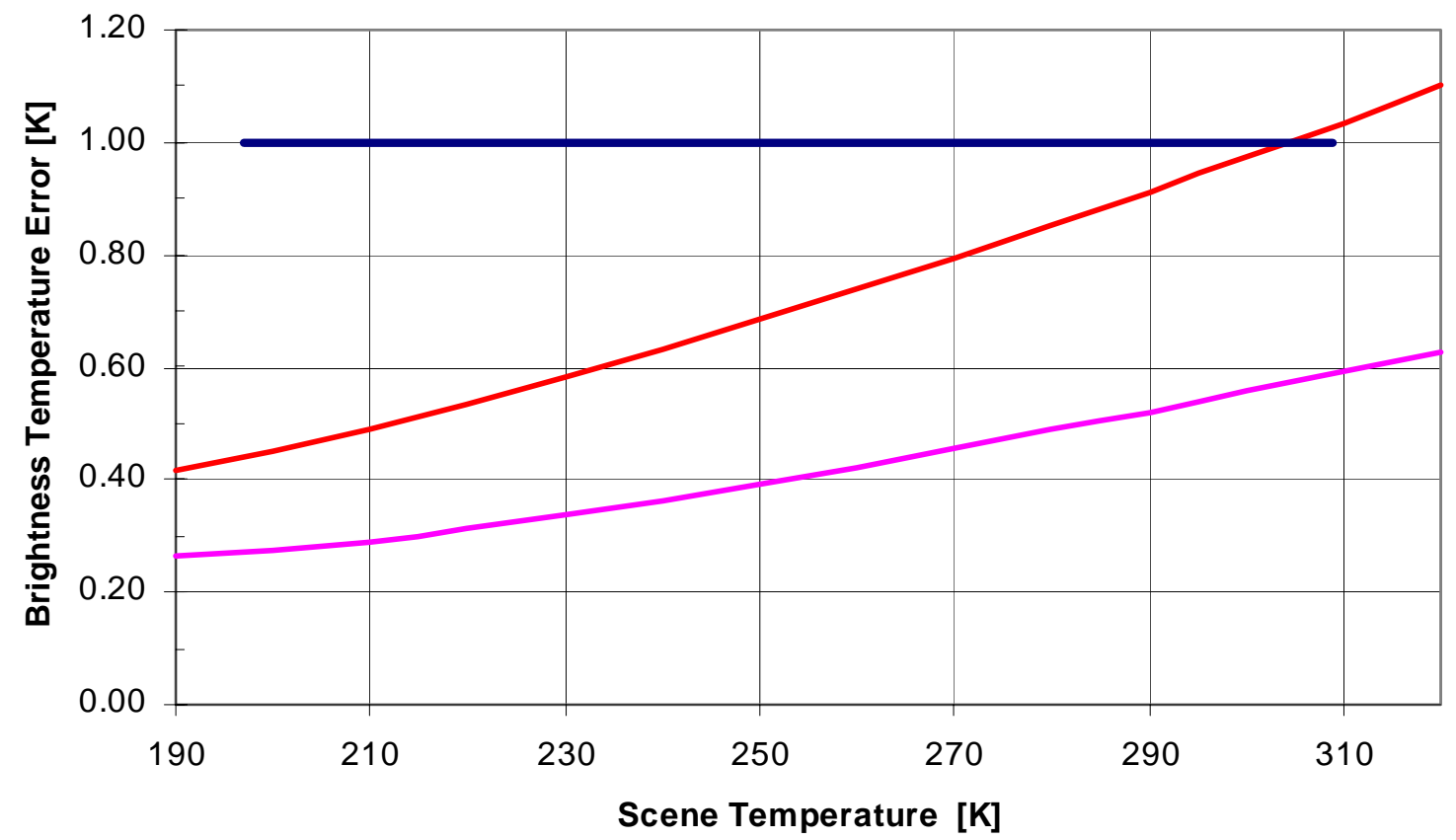

Figure 9. Uncertainty estimate (3-sigma) for the GIFTS radiometric calibration at $10 \mu \mathrm{m}$ for the blackbody contribution only (lower magenta curve) and compared to that of a lower emissivity external blackbody (middle red curve). The GIFTS requirement is $1 \mathrm{~K}$. This result shows the potential advantage of using internal high emissivity reference sources with well characterized temperature gradients over a large aperture external blackbody that is subject to large temperature forcing.

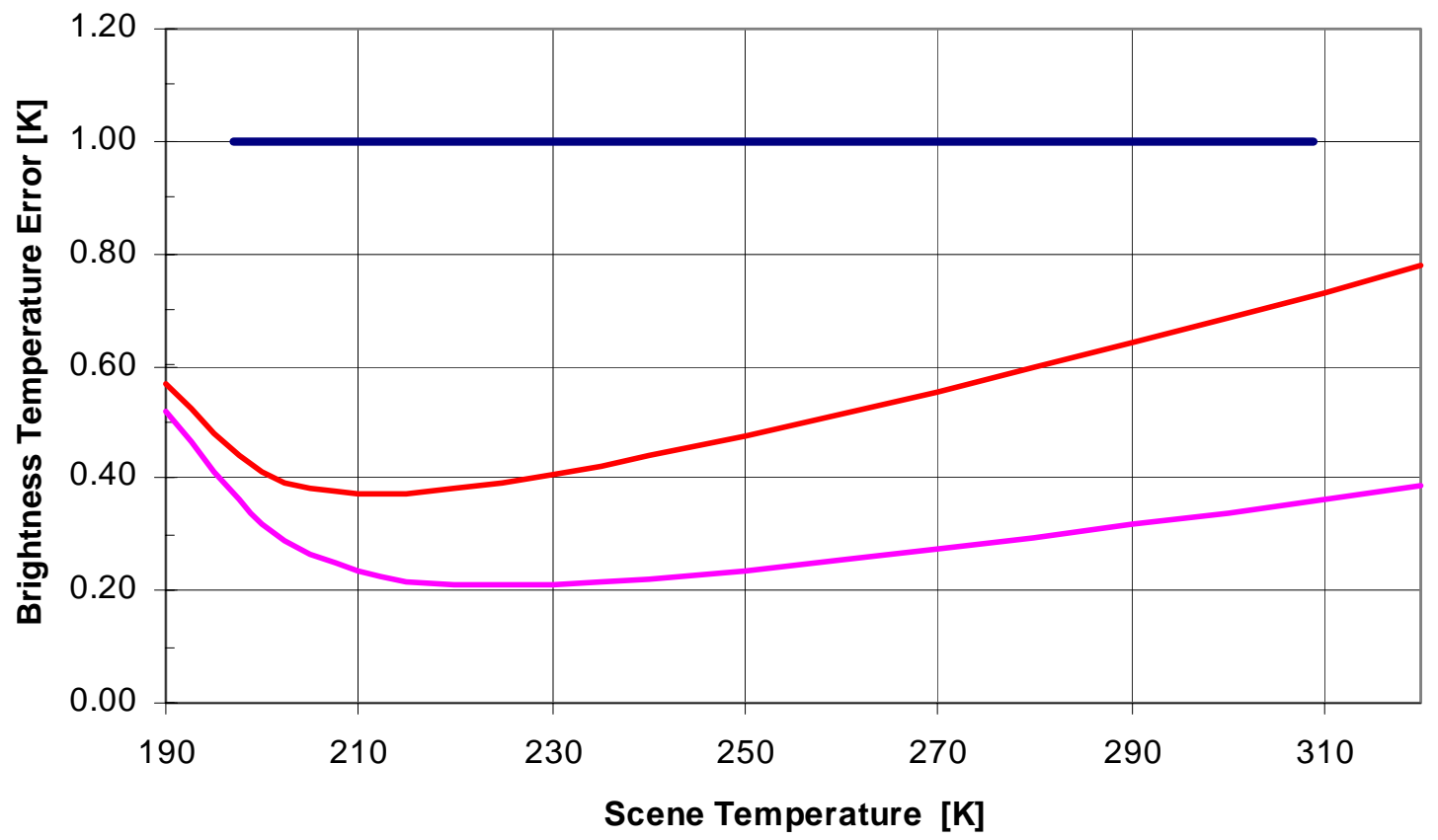

Figure 10. Same as Fig. 9 but for $5 \mu \mathrm{m}$.

Copyright 2004 Society of Photo-Optical Instrumentation Engineers.

This paper was published in the proceedings of the SPIE, Fourth International Asia-Pacific Environmental Remote Sensing Symposium, 8 November 2004; Honolulu, Hawaii, and is made available as an electronic preprint with permission of SPIE. One print may be made for personal use only. Systematic or multiple reproduction, distribution to multiple locations via electronic or other means, duplication of any material in this paper for a fee or for commercial purposes, or modification of the content of the paper are prohibited. 


\section{GIFTS SPECTRAL CALIBRATION}

The GIFTS itself is a fundamental spectral standard, due to the use of a stable laser reference coupled with the FTS design (i.e. independent of hard to characterize optical properties like blur that mandate extensive Instrument Line Shape, ILS, testing in T/V). An onboard stable laser reference makes spectral calibration essentially independent of instrument temperature (simplifies T/V testing). The GIFTS Instrument Line Shape has no significant dependence on scene content (cloud non-uniformity), for the small angles involved from geo orbit (simplifies T/V testing, applications, and improves accuracy). A single-footprint grating instrument designed like AIRS has this property too, but not an imaging grating that must use the detector array as the exit slit. The short and long term geometric and laser frequency stability, and the resulting spectral calibration, of GIFTS are expected to be very good by design (better than requirement). Spectral calibrations with Earth scene data will be used to establish and monitor the onorbit calibration (consistency with T/V characterization is expected) ${ }^{13}$. Spectral positions of selected atmospheric lines are known with high accuracy and are used to determine the calibration ${ }^{14}$. For a given clear sky Earth spectrum, the effective laser wavenumber and resulting wavenumber scale of the observed spectrum is varied to produce best agreement with a calculated spectrum (1 ppm capability has been proven with S-HIS observations and coincident in situ observations of temperature and water vapor).

The contribution to the total calibration budget caused by spectral calibration uncertainties is given in Fig. 11. The requirement for absolute calibration is $<5 \mathrm{ppm}$ (parts per million). Since we believe this requirement is too loose we have assigned an error budget of $<1 \mathrm{ppm}$ and have made an engineering best estimate on the absolute knowledge that is better than $0.3 \mathrm{ppm}$. The contributions to this absolute error estimate have been identified as coming mainly from the on-orbit determination using the positions of known atmospheric absorption lines, e.g. carbon dioxide.

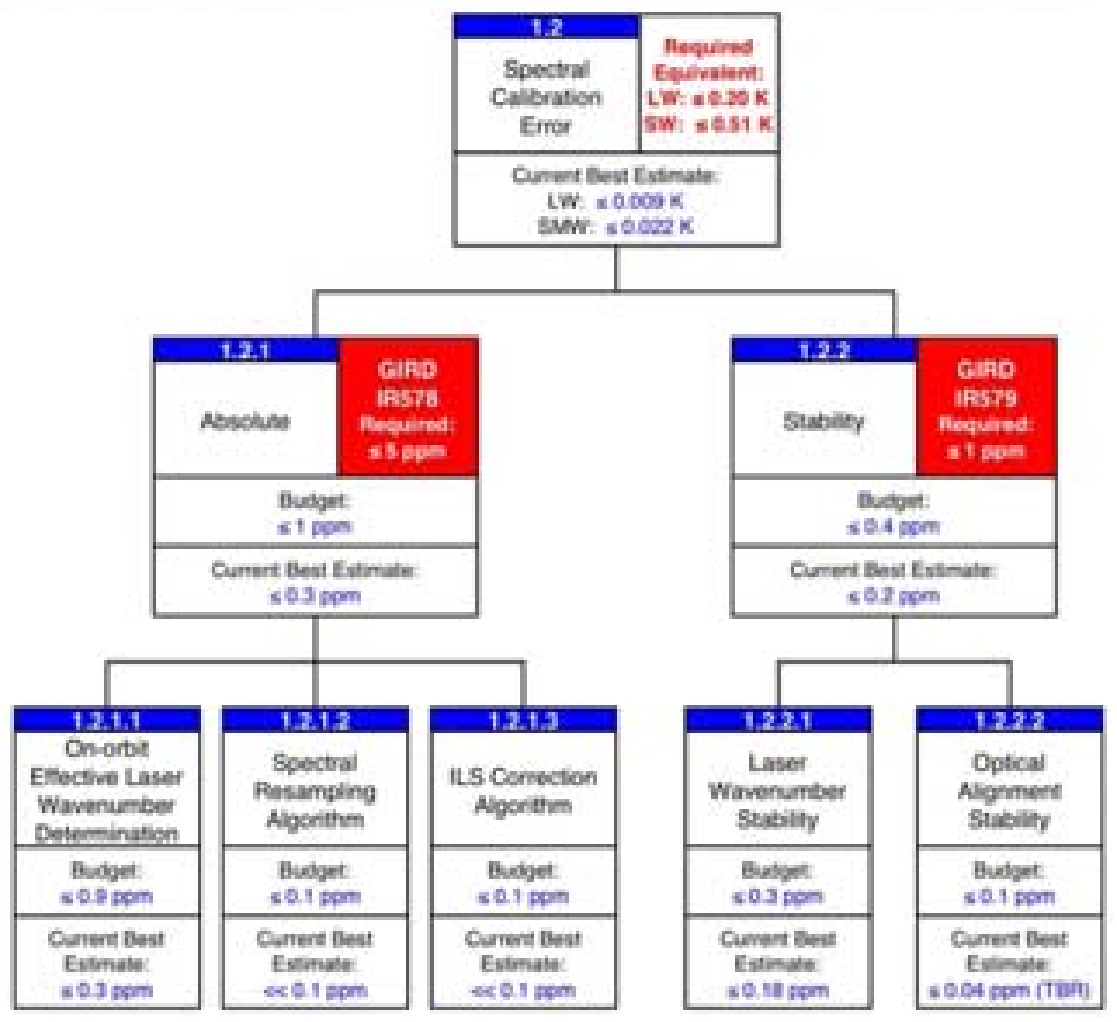

Figure 11. GIFTS spectral calibration budget and engineering best estimates. The spectral error budget is much tighter than the requirement in order to prevent errors in spectral knowledge from providing a significant contribution to the total calibration error budget. This is possible since the GIFTS makes use of a stable on-board laser combined with the FTS design which makes determination of the absolute spectral knowledge possible during flight using atmospheric absorption lines as a spectral reference. This approach has been demonstrated with the UW-SSEC Scanning-HIS aircraft instrument.

Copyright 2004 Society of Photo-Optical Instrumentation Engineers.

This paper was published in the proceedings of the SPIE, Fourth International Asia-Pacific Environmental Remote Sensing Symposium, 8 November 2004; Honolulu, Hawaii, and is made available as an electronic preprint with permission of SPIE. One print may be made for personal use only. Systematic or multiple reproduction, distribution to multiple locations via electronic or other means, duplication of any material in this paper for a fee or for commercial purposes, or modification of the content of the paper are prohibited. 
Table 1. Calibration uncertainty analysis parameters.

\begin{tabular}{|c|c|c|}
\hline \multicolumn{3}{|c|}{ Input Parameters } \\
\hline wn & Wavenumber & See figures. \\
\hline tau & $\begin{array}{lr}\text { Telescope (2) } & \text { elements and } \\
\text { blackbody } & \text { mirror } \\
\text { transmission } & \end{array}$ & 0.913 \\
\hline Thbb & Hot blackbody temperature & $300 \mathrm{~K}$ \\
\hline Tcbb & Cold blackbody temperature & $265 \mathrm{~K}$ \\
\hline Tspace & Temperature of space & $4 \mathrm{~K}$ \\
\hline Ttel & Telescope temperature & $265 \mathrm{~K}$ \\
\hline Tstr & $\begin{array}{l}\text { Temperature of structure } \\
\text { reflecting into BB's }\end{array}$ & 265 \\
\hline Ehbb & Emissivity of hot blackbody & 0.996 \\
\hline Ecbb & Emissivity of cold blackbody & 0.996 \\
\hline \multicolumn{3}{|c|}{ Parameters Used For Temperature Stability } \\
\hline Etel & Telescope emissivity & 0.087 \\
\hline TauTot & $\begin{array}{l}\text { Total transmission through } \\
\text { instrument }\end{array}$ & 0.205 \\
\hline Ttel $\Delta$ & $\begin{array}{l}\text { Change in telescope temp } \\
\text { between earth and space } \\
\text { views }\end{array}$ & $0.5 \mathrm{~K}$ \\
\hline \multicolumn{3}{|c|}{ Uncertainty Magnitudes } \\
\hline$\Delta$ Thbb & $0.07 \mathrm{~K}$ & \\
\hline$\Delta \mathrm{Tcbb}$ & $0.07 \mathrm{~K}$ & \\
\hline$\Delta$ Ehbb & $0.002 \mathrm{~K}$ & \\
\hline$\Delta$ Ecbb & $0.002 \mathrm{~K}$ & \\
\hline$\Delta$ Tstr & $5 \mathrm{~K}$ & \\
\hline$\Delta$ tau & 0.0086 RSS & \\
\hline$\Delta$ Ttel & $2 \mathrm{~K}$ & \\
\hline
\end{tabular}

\section{SUMMARY}

This paper represents the state of the GIFTS radiometric and spectral calibration at the time of the sensor critical design review. The on-orbit calibration approach using two internal calibration references sources and a space view are estimated to meet the GIFTS requirements and to show superior performance to a single external large aperture blackbody reference. Similarly, the on-orbit absolute spectral calibration and stability is estimated to meet the design goal of minimizing the contribution of spectral calibration error to the overall calibration budget. Future work will include the verification of the engineering best estimates to the maximum extent possible during GIFTS thermal vacuum testing.

\section{ACKNOWLEDGEMENTS}

This work was supported by federal grant NAS1-00072.

\section{REFERENCES}

1. Dittberner, G. J., James J. Gurka, and Roger W. Heymann, 2003: “NOAA’s GOES satellite program—status and plans" 19th Conference on IIPS, 83rd Annual Meeting, 8-13 February 2003, Long Beach, CA. Published by the American Meteorological Society, Boston, Mass.

2. Gurka, J. J., Gerald J. Dittberner, Pamela Taylor, and Timothy J. Schmit, 2003: "Specifying the requirements for imaging and sounding capabilities on the GOES-R series" 12th Conference on Satellite Meteorology and

Copyright 2004 Society of Photo-Optical Instrumentation Engineers.

This paper was published in the proceedings of the SPIE, Fourth International Asia-Pacific Environmental Remote Sensing Symposium, 8 November 2004; Honolulu, Hawaii, and is made available as an electronic preprint with permission of SPIE. One print may be made for personal use only. Systematic or multiple reproduction, distribution to multiple locations via electronic or other means, duplication of any material in this paper for a fee or for commercial purposes, or modification of the content of the paper are prohibited. 
Oceanography, 83rd Annual Meeting, 8-13 February 2003, Long Beach, CA. Published by the American Meteorological Society, Boston, Mass.

3. Revercomb, H. E., H. Buijs, H. B. Howell, D.D. LaPorte, W. L. Smith, and L. A. Sromovsky, 1988: "Radiometric calibration of IR Fourier Transform Spectrometers: solution to a problem with the High Resolution Interferometer Sounder", Appl. Opt., 27, 3210-3218.

4. Smith, W. L., D. K. Zhou, F. W. Harrison, H. E. Revercomb, A. M. Larar, A. H. Huang, B. Huang, 2000: "Hyperspectral remote sensing of atmospheric profiles from satellites and aircraft" presented at SPIE's Second International Asia-Pacific Symposium on Remote Sensing of the Atmosphere, Environment, and Space, Sendai, Japan, 9-12 October 2000.

5. Knuteson R. O., H. E. Revercomb, F. A. Best, N. C. Ciganovich, R. G. Dedecker, T. P. Dirkx, S. C. Ellington, W. F. Feltz, R. K. Garcia, H. B. Howell, W. L. Smith, J. F. Short, D. C. Tobin, 2004: "Atmospheric Emitted Radiance Interferometer (AERI) Part I: Instrument Design” J. Atmos. Oceanic Technol (in press).

6. Knuteson R. O., H. E. Revercomb, F. A. Best, N. C. Ciganovich, R. G. Dedecker, T. P. Dirkx, S. C. Ellington, W. F. Feltz, R. K. Garcia, H. B. Howell, W. L. Smith, J. F. Short, D. C. Tobin, 2004: “Atmospheric Emitted Radiance Interferometer (AERI) Part II: Instrument Performance” J. Atmos. Oceanic Technol (in press).

7. Revercomb, H.E., et al., 1998: "Recent results from two new aircraft-based Fourier transform interferometers: The Scanning High-resolution Interferometer Sounder and the NPOESS Atmospheric Sounder Testbed Interferometer" 8th International Workshop on Atmospheric Science from Space using Fourier Transform Spectrometry (ASSFTS), Toulouse, France, 16-18 November 1998.

8. Goody, R. and R. Haskins, 1998: “Calibration of Radiances from Space” J Climate, 11, 754-758, 1998.

9. Best, F. A., H. E. Revercomb, R. O. Knuteson, D. C. Tobin, S. D. Ellington, M. W. Werner, D. P. Adler, R. K. Garcia, J. K. Taylor, N. N. Ciganovich, W. L. Smith, G. E. Bingham, J. D. Elwell, and D. K. Scott, 2004: "The Geosynchronous Imaging Fourier Transform Spectrometer (GIFTS) On-board Blackbody Calibration System” In: Proceedings of the SPIE, Fourth International Asia-Pacific Environmental Remote Sensing Symposium, 8 November 2004, Honolulu, Hawaii.

10. Elwell, J. D., D. K. Scott, H. E. Revercomb, F. A. Best, R. O. Knuteson, 2003 : “An overview of Ground and On-orbit Characterization and Calibration of the Geosynchronous Imaging Fourier Transform Spectrometer (GIFTS)" in Proceedings of CALCON 2003, Characterization and Radiometric Calibration for Remote Sensing, Space Dynamics Laboratory / Utah State University, Logan, Utah, 15-18 September 2003.

11. Best, F. A., H. E. Revercomb, G. E. Bingham, R. O. Knuteson, D. C. Tobin, D. D. LaPorte, and W. L. Smith, 2000: "Calibration of the Geostationary Imaging Fourier Transform Spectrometer (GIFTS)" presented at SPIE's Second International Asia-Pacific Symposium on Remote Sensing of the Atmosphere, Environment, and Space, Sendai, Japan, 9-12 October 2000.

12 Bingham, G. E., R. J. Huppi, H. E. Revercomb, W. L. Smith, F. W. Harrison, 2000: "A Geostationary Imaging Fourier Transform Spectrometer (GIFTS) for hyperspectral atmospheric remote sensing” presented at SPIE's Second International Asia-Pacific Symposium on Remote Sensing of the Atmosphere, Environment, and Space, Sendai, Japan, 9-12 October 2000.

13. Tobin, D. C., H. E. Revercomb, R. O. Knuteson, 2003: “On-orbit Spectral Calibration of the Geosynchronous Imaging Fourier Transform Spectrometer (GIFTS)” in Proceedings of CALCON 2003, Characterization and Radiometric Calibration for Remote Sensing, Space Dynamics Laboratory / Utah State University, Logan, Utah, 15-18 September 2003.

14. Rothman, L. S., et al., 1998: “The HITRAN Molecular Spectroscopic Database and HAWKS” 1996 Edition, Journal of Quantitative Spectroscopy and Radiative Transfer, 60, pp. 665-710.

Copyright 2004 Society of Photo-Optical Instrumentation Engineers.

This paper was published in the proceedings of the SPIE, Fourth International Asia-Pacific Environmental Remote Sensing Symposium, 8 November 2004; Honolulu, Hawaii, and is made available as an electronic preprint with permission of SPIE. One print may be made for personal use only. Systematic or multiple reproduction, distribution to multiple locations via electronic or other means, duplication of any material in this paper for a fee or for commercial purposes, or modification of the content of the paper are prohibited. 